\title{
Jeunesse et plage : approche sociolinguistique des publicités contemporaines
}

\author{
Abraham, Marine \\ Universidad de Murcia, marine.abraham1@um.es
}

\begin{abstract}
Resumen
En el presente estudio nuestro objetivo será revelar de qué manera los profesionales de la publicidad emplean alegorías haciendo referencia al agua del mar con el fin de convencer a sus futuros clientes de consumir el producto anunciado. Centraremos nuestra investigación en una campaña situada en una zona costera que pone en escena dos adolescentes, población que representa otro mito evidente de nuestras sociedades occidentales. Daremos cuenta de la semiótica de las propagandas contemporáneas acorde con los aspectos lingüísticos anclados en el mundo de los jóvenes. De esta manera pondremos en evidencia que los profesionales de la publicidad disponen de las realidades sociolingüísticas adolescentes y que ponen en escena una selección de sus preocupaciones en un universo idílico. En definitiva, demostraremos que la combinación del elemento agua y del factor juventud resulta ser óptimo para las campañas publicitarias modernas.
\end{abstract}

Palabras clave : playa ; adolescente ; spot publicitario ; sociolingüística ; alegoría.

\section{Résumé}

Lors de la présente étude, notre objectif sera de révéler de quelle manière les professionnels de la publicité emploient les allégories se référant à l'eau de mer afin de convaincre leurs futurs clients de consommer le produit objet de la publicité. Nous centrerons nos recherches sur une campagne située en zone balnéaire mettant en scène deux jeunes d'aujourd'hui, population représentant un autre mythe manifeste de nos sociétés occidentales. Nous rendrons compte de la sémiotique des propagandes contemporaines en accord avec des aspects linguistiques ancrés dans le monde des jeunes. Nous mettrons ainsi en évidence le fait que les professionnels de la publicité disposent des réalités sociolinguistiques adolescentes et qu'ils mettent en scène une sélection de leurs préoccupations environnantes dans un univers idyllique. En définitive, nous démontrerons que la combinaison de l'élément eau et du facteur jeune s'avère être judicieux dans les campagnes publicitaires modernes.

Mots-clés : plage ; adolescent ; spot publicitaire ; sociolinguistique ; allégorie.

\begin{abstract}
This research paper aims to reveal the way in which the advertisers use allegories related to water in order to convince future customers to buy the advertised product. We will focus our research on a campaign which takes place in a seaside zone and stages two young people who represent an-other evident myth of our Western societies. We will report on the semiotics of contemporary propaganda in agreement with linguistic aspects embedded in the teenage world. As such, we will highlight the fact that advertisers have teenage sociolinguistics realities at their disposal and stage a selection of their concerns in an idyllic universe. Finally, we will demonstrate that the combination of the element, water, and the factor of youth prove to be successful in modern campaigns.
\end{abstract}

Keywords : beach : teenager : commercial : sociolinguistic : allegory. 


\section{Introduction}

L'emploi des images de l'eau dans les spots ou affiches actuels n'est pas anodin. En effet, elle symbolise un temps de rupture et d'évasion, des moments jouissifs dont tout individu souhaite profiter. La plage transmet ainsi des émotions positives, ce qui permet d'une part de capter l'attention des consommateurs et, d'autre part, de formater leurs sentiments envers le produit ou la marque. La publicité réussit de cette manière à rendre matérielles des notions abstraites telles que la liberté, idéal qui semble désormais pouvoir s’acheter à travers la consommation. Associée à la figure du jeune, la campagne publicitaire s'inscrivant en bord de mer n'en est que plus attirante. De ce fait, la jeunesse représente en quelque sorte l'âge idéal pour tout type de public, tant chez les enfants qui désirent accéder à cette vie d'apparence autonome que chez les adultes qui rêvent de jeunesse éternelle. Nous appuierons nos allégations sur un spot publicitaire du yaourt à boire de la marque française Yop datant de 2013 qui révèle parfaitement la symbiose de la mer et de l'adolescent en publicité ${ }^{1}$.

\section{Jeunesse et eau : deux mythes publicitaires}

\subsection{L'adolescent : acteur principal des médias}

Nous remarquons ces dernières années l'intérêt grandissant des médias pour le public adolescent. En effet, cette période de vie caractérisée par des changements le plus souvent complexes mais nécessaires au développement physique et psychique de l'individu n'est pas sans séduire les moyens de communication de nos sociétés contemporaines. Dans le cas de notre étude se centrant sur la publicité, il est évident que les cibles sont variées et dépendent du produit ou de la marque objet de la campagne publicitaire. Cependant, il semble que les jeunes sont dans la plupart des cas les acteurs de campagnes publicitaires dirigées à tout type de public. Nous nous rendons ainsi compte que même si le produit n'est pas exclusivement destiné aux adolescents, ils en seront l'image. Auparavant, la femme au foyer était le centre de l'activité publicitaire, puis la famille traditionnelle (Jean et al., 2014) avec la mise en scène d’une situation familiale idéale. Mais comme le signale León, ces modèles d'exemplarité ont laissé place à l'archétype jeune :

La pubertad resulta sin duda la edad privilegiada en el plano de la mensajería social, siendo los jóvenes protagonistas indiscutibles de la publicidad, del cine y la televisión, y una referencia ideal para el resto de edades, un modelo tanto para los niños como para los adultos, los primeros porque quieren llegar rápido al futuro, los segundos porque desean una prolongación del pasado, alimentando a su adolescente interior, ajeno al tiempo ${ }^{2}$ (2001 : 111).

D’un côté, les enfants idéalisent les adolescents : ils les perçoivent comme étant des individus autonomes sans pour autant assumer de fortes responsabilités ; ce sont pour eux des modèles de liberté puisqu'ils paraissent ne plus suivre à la lettre les directives de leurs parents et ne plus répondre aussi docilement aux normes sociétales. D’un autre côté, les adultes, submergés d'obligations tant professionnelles que familiales et financières, se remémorent une jeunesse souvent magnifiée et espèrent perpétuer ce temps passé à travers par exemple leurs habitudes de consommation. Bernheim souligne que les individus souhaitent désormais « vieillir jeune » (2004:238) or nous sommes à même de constater que le «mythe de la jeunesse éternelle» (Ibid. : 239) se trouve immortalisé dans tous les moyens de communication environnants. Les enfants comme les adultes ont donc une représentation idyllique de la jeunesse : les adolescents sont le symbole de l'espoir, l'insouciance, la beauté, l'énergie, la vivacité, l'avenir, la nouveauté, l'innovation et tant d'autres facettes positives. Ils représentent finalement des aspects heureux et incarnent la liberté, c'est pourquoi les professionnels de la publicité en font leur principaux protagonistes.

Dans le spot publicitaire pour la marque Yop, nous retrouvons la plupart des caractéristiques citées précédemment. À sa vue, l'enfant enviera le jeune protagoniste qui a la possibilité de se retrouver seul - sans l'autorité parentale - sur une plage mais accompagné d'une jeune fille, alors que l'adulte jalousera cette relation naissante où l'individu n'est pas encore

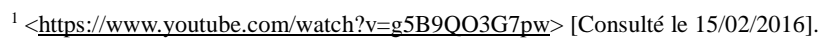

${ }^{2}$ La puberté demeure sans aucun doute l’âge privilégié sur le plan de la messagerie sociale, les jeunes étant les acteurs principaux de la publicité, du cinéma et de la télévision, et une référence idéale pour le reste des âges, un modèle tant pour les enfants que pour les adultes, les premiers parce qu'ils souhaitent accéder rapidement au futur, les seconds car ils désirent une prolongation du passé, en alimentant leur adolescent intérieur, étranger au temps. (traduction personnelle) 
engagé sentimentalement. L’insouciance émanant de cette vidéo publicitaire et, à la fois, le pouvoir de décision dont fait preuve l'adolescent permettent de capter l'attention tant des jeunes téléspectateurs que celle des plus âgés.

\subsection{L'eau : symbole de liberté}

Tout comme la jeunesse, nous verrons que l'eau manifeste également des valeurs sociétales positives : un sentiment de liberté émane effectivement de ces deux facteurs qui fascinent ainsi les concepteurs-rédacteurs de publicités contemporaines.

Nous nous devons dans un premier temps de souligner l'importance de la plage tant par le passé que de nos jours : Lageiste évoque ainsi dans son écrit que l'eau s’avère être une source de plaisir depuis toujours et que « la plage est un phénomène sociétal durable dont les pratiques n’ont eu cesse d'évoluer » (2008: 15). La plage représente en effet un lieu plaisant, et depuis quelques décennies, une destination touristique incontournable (Lazzarotti, 2012) pour toutes les classes sociales. Autrefois réservée à une élite, les plages se sont de ce fait démocratisées au $\mathrm{XX}^{\mathrm{e}}$ siècle. Il est toutefois primordial de différencier la plage familiale, généralement surpeuplée, de la plage que l’on pourrait nommer sauvage, laissant de côté les plages privées destinées à un public bien spécifique. Habituellement difficile d'accès, la plage déserte et non familiale évoque le romantisme, le mystère, le secret et l'interdit pour les adolescents, comme nous le démontre notre spot publicitaire, alors que la plage familiale nous suggère d'autres aspects tels que des retrouvailles en famille, un lieu bruyant et chaotique, un manque d'intimité conséquent, laissant place à une promiscuité pittoresque, etc. La plage symbolise donc différentes émotions qui seront cependant toujours heureuses.

Dans un second temps, Lageiste émet une autre idée en relation directe avec les espaces hétérotopiques décrits par Foucault (1984) : la plage retrace selon ce premier « un espace qui n’a jamais laissé les hommes indifférents et dont le pouvoir de fascination semble tenir au fait qu'il soit en rupture avec les autres espaces » (2008: 7). Cet auteur met donc en évidence l'existence d'une étroite relation entre lieu de rupture et objet de convoitise. Si nous prenons en considération la définition que donne Foucault des hétérotopies, « des sortes de lieux qui sont hors de tous les lieux, bien que pourtant ils soient effectivement localisables » (1984: 47), nous constatons que la plage représente bel et bien un espace physique en rupture avec la vie quotidienne des individus de nos sociétés contemporaines, puisque, comme mentionné précédemment, c'est avant tout un lieu de vacances, un espace où les individus passent leur temps libre qui se trouve totalement en décalage avec les endroits qu'ils fréquentent habituellement tels que leur lieu de travail ou de formation ou leur espace de vie.

Rappelons que les plages sont des emplacements géographiques réels qui ne peuvent être utopiques. Toutefois, elles ont une part de fiction non négligeable, ce qui les rend hétérotopiques. Sabot précise quelque peu les affirmations de Foucault en décrivant les hétérotopies comme étant « des emplacements situés dans l’espace général de notre expérience, affectés donc d'une certaine réalité matérielle, mais creusant aussi l'évidence de l'espace vécu jusqu'à en contester l'usage ordinaire » (2012 : 25). La publicité dont nous faisons ici l'étude dépeint parfaitement cette perspective imaginaire au sein d'un lieu concret et authentique. Les deux jeunes ne sont en aucun cas allongés sur la plage à profiter d'un bain de soleil ou en train de se baigner, ils paraissent plutôt en train de flirter à l'abri des regards indésirables. Pour corroborer la caractéristique hétérotopique de ce spot publicitaire, nous nous attarderons sur le quatrième principe développé par Foucault explicitant que «les hétérotopies sont liées, le plus souvent, à des découpages du temps, c'est-à-dire qu'elles ouvrent sur ce qu'on pourrait appeler, par pure symétrie, des hétérochronies ; l'hétérotopie se met à fonctionner à plein lorsque les hommes se trouvent dans une sorte de rupture absolue avec leur temps traditionnel » (1984 : 48). Dans le cas des adolescents, figures de cette campagne publicitaire, la rupture temporelle ne peut être contestée : ils partagent un moment supposé être idyllique en un lieu paradisiaque au lieu de se trouver dans leur milieu coutumier. Nous nous confrontons ici à une interruption de leur temps académique, familial ou amical qui représente pourtant leur routine quotidienne. La plage est finalement pour eux un lieu qui laisse place à une parenthèse de vie bucolique, presque hors du réel.

L'eau reflète par conséquent des émotions positives et est symbole de circonstances agréables. Elle est synonyme de bonheur, d'échappatoire à la réalité, de temps de rupture et d'évasion, de jouissances, de vacances, etc. On attribue d'ailleurs une importance considérable aux paysages de bord de mer pour les impressions qu'ils procurent à leur contemplation : la plage stimule en effet l'imaginaire de chacun, fait naître ou ressurgir de fortes émotions, elle permet 
l'évasion, le rêve éveillé et l'accès à une certaine liberté. Pour des raisons diverses mais non pas incompatibles, la plage comme les jeunes représentent deux aspects réels de nos vies modernes qui fascinent et excitent l'imaginaire des individus. La vidéo publicitaire étudiée met en scène ces deux éléments ici complémentaires et atteint les objectifs visés par tout publiciste : elle capte l'attention d'un public jeune et moins jeune et fait naître en lui un désir de consommer ce produit qui signifie bien plus qu'une manière de s'alimenter puisqu'il promeut un comportement libre à travers ces personnages adolescents se retrouvant seuls sur une plage déserte.

\section{La publicité : tableau fictif des réalités adolescentes}

\subsection{Matérialisation de notions abstraites}

Nous terminions le paragraphe précédent évoquant le pouvoir fantasmagorique de la plage et le rôle non moins mythique des jeunes protagonistes dans les publicités actuelles :

L'imaginaire de la plage renvoie invariablement à des représentations de vastes vides où l'on se trouve seul à goûter les plaisirs sensoriels de l'instant présent. Cette image du paradis terrestre empruntée aux plages polynésiennes - sable banc, cocotiers et mer turquoise -, largement diffusée et exploitée par les professionnels du marketing publicitaire du tourisme, s’oppose pourtant à la réalité. Le quotidien estival des plages les plus courues se trouve surpeuplé (Lageiste, $2008: 19)$.

Il est primordial de mentionner ici le cas encore plus démonstratif des adolescents : en effet, les milieux balnéaires les plus fréquentés et accessibles aux jeunes sont généralement les plages familiales en raison de leur facilité d’accès. Les adolescents ne disposant pas de moyen de transport propre, si ce n’est éventuellement d'un deux roues, ils sont donc souvent dépendants du véhicule de leurs parents ou des transports en commun. C’est pourquoi ils se retrouvent d'ordinaire sur les plages qui s'avèrent réellement à la portée de tous. Nous sommes donc confrontés à un contraste éloquent entre les plages de notre vie quotidienne et celles des campagnes publicitaires, tel que nous le montre le court-métrage faisant la publicité du yaourt liquide Yop où les deux jeunes gens se retrouvent seuls sur une plage déserte qui apparaît comme étant difficile à atteindre.

Nous devons désormais nous interroger sur la nature des mécanismes psycho-sociaux mis en place qui permettent la stimulation de la veine consommatrice de chacun. Dans son ouvrage Le bonheur conforme, Brune critique le fait que « toutes les dimensions de la vie vont pouvoir s’épuiser dans la seule consommation » (1985 : 62-63) et reprend ainsi les affirmations de Baudrillard qui révélait en 1970 que la grande majorité des populations occidentales vivent à travers la consommation et qu'elles sont persuadées d'être ce qu'elles consomment. En d'autres termes, la plupart des individus sont aujourd'hui convaincus que l'achat de produits et services délimitera leur identité individuelle et sociale. Cette détermination commence dès le plus jeune âge compte tenu de la société consumériste dans laquelle nous nous développons. Les publicitaires tentent par exemple de convaincre les adolescents que l'acquisition de certaines marchandises leur permettra cette autonomie tant convoitée : «ils associent à la consommation les valeurs adolescentes que leurs enquêtes ont pu déceler : la sociabilité, la tolérance et l'autonomie, le but étant de persuader que s'émanciper c’est consommer » (Coslin, 2007 : 159-160). C’est ainsi le cas de la publicité pour Yop dans laquelle le jeune est perçu comme une personne pouvant faire ses propres choix et où les parents ne sont pas représentés ou même évoqués. Elle va même plus loin en représentant l'adolescent partageant un moment intime avec une jeune fille, symbole d'émancipation sociale et sexuelle pour cette tranche d’âge. Le produit, bien que présent tout au long du spot, reste secondaire jusqu'aux dernières secondes de la vidéo. Tout est fait pour que le téléspectateur se concentre sur la situation amoureuse vécue sur une plage idyllique, même la musique de fond, représentative de toute une génération. En effet, la chanson Unbreakable du boys band irlandais à succès Westlife date de 2002 et quoique le texte ait été fortement coupé pour les besoins de la publicité, les quelques paroles audibles sont facilement reconnaissables et accroissent le caractère romantico-kitsch du spot publicitaire sur lequel le récepteur doit s’attarder ( $c$. analyse des paroles dans la partie 3.1 de cet article). La publicité vend du rêve jusqu'au dénouement humoristique renvoyant lui aussi à la position forte du jeune homme qui, même à l'âge pubère, est capable de refuser les avances d'une jeune fille objet de convoitise de tout adolescent. 
Comme le signale Ortega (2004), la publicité rend donc matérielles des notions abstraites. La fonctionnalité des objets en soi ayant peu à peu disparu, les campagnes se fondent désormais sur l'émotion (Eguizábal, 2007). Non seulement elles cherchent à produire des sentiments positifs et heureux chez l'individu mais elles ont également pour but de transformer un produit, tel que le yaourt liquide ici, en marchandise indispensable à l'autonomie et à la satisfaction du jeune.

\subsection{Sélection choisie des préoccupations adolescentes}

Comme nous le signale très justement Eguizábal dans son écrit sur l’histoire récente de la publicité, « la praxis publicitaria trabaja desde la "realidad social”, sus mensajes constituyen algo así como un gran retrato de lo social ${ }^{3}$ » (2009 : 10). Les spécialistes de la création publicitaire s’imprègnent ainsi de la réalité et du quotidien des adolescents afin de créer leurs campagnes, ils analysent leur vie dans le but de déchiffrer leurs sujets de prédilection, tout comme le font les entrepreneurs lorsqu’il s’agit de dégager les produits qu'affectionnent les plus jeunes. La publicité est finalement le portrait des préoccupations adolescentes, les publicistes les mettant en scène de manière ingénieuse. Afin de retranscrire au mieux leurs goûts et leurs préoccupations, nous avons mené une enquête dans divers établissements français. Ces questionnaires collectés en début d’année 2013 nous ont entre autres permis de déterminer quelles étaient les préférences adolescentes en matière de consommation et à quoi s’intéressaient réellement les jeunes de nos sociétés occidentales. Nous remarquons que plusieurs des thèmes abordés dans le spot publicitaire étudié sont représentatifs des intérêts grandissants à cet âge précis : l’industrie agro-alimentaire (le produit en lui-même), l'émancipation physique et psychologique ainsi que les relations amoureuses. Ce sera ce dernier paramètre qui nous intéressera tout particulièrement puisque c'est ce qui finalement retient le plus l'attention du récepteur de la publicité : les rapports qu'entretient l'adolescent avec sa jeune prétendante.

Le développement psycho-sexuel de l'adolescent demeure en effet nécessaire à son entrée dans la vie adulte, tout comme le sont ses développements cognitif et moral. Premièrement, « les réalités amoureuses s’imposent à la conscience et s'inscrivent dans l'expérience de l'adolescence. L'engagement progressif dans une relation intime avec un partenaire constitue une des tâches développementales de l'adolescence » (Claes, 2003 : 112). Il est en effet question d'un passage obligé pour que tout individu ait une vie adulte équilibrée. La découverte de son propre corps et la reconnaissance de ceux d'autrui sont deux éléments primordiaux qui doivent avoir lieu durant la phase pubère de l'individu. Il en est de même pour l'exploration psychique qui permettra l'entendement du jeune avec les individus qui l'entourent tout au long de sa vie. En second lieu, bien plus que la découverte en elle-même, ce serait l'épanouissement sexuel qui, selon Freud (1905, 1917, 1923), se révélerait être indispensable à la construction identitaire de l’individu. En effet, la consolidation du Moi que rendra possible cette maturité libidinale occasionnera la création de l'identité individuelle et sociale de l'individu. Néanmoins, ne confondons point maturité sexuelle avec passage à l'acte entre deux personnes. L'auteur entend par épanouissement intime le fait de prendre du plaisir en se découvrant physiquement et en comprenant les aspects psychosexuels des personnes de notre entourage.

Enfin, il faut reconnaître que les moyens de communication vendent l'amour comme une circonstance nécessaire à la plénitude personnelle et sociale :

Cette valorisation de l'amour a toujours cours dans la société contemporaine et le thème des amours heureuses ou malheureuses loge constamment au cœur des œuvres artistiques les plus marquantes, qu'il s'agisse de littérature, de peinture, de sculpture, de musique, de chansons ou de films. En exaltant de façon persistante le thème de la passion amoureuse, notre société propose des modèles d'existence et dessine des scripts de vie où l'amour occupe une place majeure comme condition d'une vie réussie et heureuse, au point que nos contemporains estiment que leur bonheur dépend largement de leur capacité de créer et d'entretenir des liens amoureux (Claes, 2003 : 123-124).

Dans le spot publicitaire analysé, nous retrouvons un adolescent qui paraît indépendant émotionnellement et sûr de lui, préférant son Yop à sa prétendante. Nous faisons face à un décalage surprenant à forte teneur humoristique. Cette annonce semble donc transgresser les codes normés des relations amoureuses : nous pourrions en effet croire que l'histoire

${ }^{3}$ La praxis publicitaire travaille depuis la réalité sociale, ses messages constituent en quelque sorte un grand portrait de ce qui est social. (traduction personnelle) 
retranscrite dans la publicité va à l'encontre de ce que nous mentionnions précédemment mais il n'en est rien puisque le jeune protagoniste semble finalement avoir réussi une approche amoureuse. Il a simplement pris de l'assurance dans cette relation sentimentale, ce qui symbolise un développement psycho-affectif manifeste. Le scénario nous enseigne qu’il a surmonté cette première étape de l'acceptation de soi et la recherche de l'autre et qu'il se trouve désormais plus près de la maturité affective d'un adulte.

\title{
3. Un discours linguistique en accord avec les besoins psycho-sociaux des adolescents
}

Dans un premier temps nous ferons l'analyse des paroles de la chanson accompagnant le spot publicitaire objet de notre étude :

\author{
Took my hand, \\ Touched my heart, \\ Held me close, \\ Believe that you're mine, \\ This love is unbreakable, \\ This love is unbreak ${ }^{4} \ldots$
}

Cet air, qui complète les images romantiques des deux jeunes adolescents réunis sur une plage déserte, permet au récepteur de s'imprégner directement de l'histoire sentimentale que nous propose le spot publicitaire. Absolument toutes les expressions utilisées évoquent d'ailleurs la romance qui subsiste entre les deux protagonistes avec entre autres la répétition coup sur coup du terme love, mot faisant partie du refrain « this love is unbreakable ». Les concepteurs-rédacteurs insistent du reste sur cette partie de la chanson afin de valoriser le produit de la publicité qui deviendra finalement plus fort que l'amour. En effet, le deuxième refrain s'arrête franchement avant que les chanteurs n'aient pu terminer de prononcer le terme « unbreakable » afin de laisser place à la formule publicitaire propre à la marque de yaourt liquide. Cette nette interruption montre clairement que le Yop l'emporte sur l'amour, qu'il est indestructible et irremplaçable.

En second lieu, nous étudierons les dires du jeune homme servant de slogan au produit et qui marquent une coupure catégorique avec le romantisme de la chanson précédente.

T’as bu mon Yop ? Heureusement qu'j’ai checké5 . C’est mon Yop !

D’une part, nous remarquons la double répétition du nom de la marque Yop en moins de cinq secondes de spot publicitaire. Alors qu'au début de l'annonce le produit restait au second plan, la situation amoureuse étant ce sur quoi le concepteur rédacteur souhaitait que nous nous concentrions, le nom du produit ainsi que son emballage reviennent en force à la fin de l'annonce. La rupture entre la première et la deuxième partie de la publicité n’en est que plus forte, ce qui rend possible l'effet de surprise qui transforme le récit amoureux en une chronique emplie d'humour. Il est également question d'un moyen mnémotechnique notable puisque, bien que la marque soit déjà très connue en France, le fait de la répéter deux fois de suite ne fait qu’accroître la capacité de mémorisation du récepteur. De plus, cette répétition n’ayant lieu qu’à la fin du spot, le futur consommateur aura la marque en tête bien plus longtemps. Ensuite, nous observons la réitération de l'adjectif possessif «mon » à deux reprises suivi directement du nom de la marque. L'emploi de ce déterminant en publicité n’est pas vain puisqu’il fait émerger chez le récepteur un sentiment d'appartenance ; ce «je » d’identification insiste en effet sur la personne qui possède le produit, cette personne se trouvant indirectement être le destinataire de la campagne publicitaire.

\footnotetext{
${ }^{4}$ Tu as pris ma main, Tu as touché mon cœur, Tu m’as serré contre toi, Je crois que tu es mienne, Cet amour est indestructible, Cet amour est indestruc... (traduction personnelle)

${ }^{5}$ Terme traduit dans le propre spot publicitaire par le mot « vérifié ».
} 
D’autre part, nous constatons l’omniprésence du langage oral avec deux assimilations phonétiques évidentes : la première portant sur le premier pronom personnel sujet «tu » qui devient «t' » au contact de la voyelle qui le suit et la seconde concernant le pronom relatif « que » qui précède le pronom personnel sujet «j’ ». La modification phonétique dans les deux cas revient à la suppression incorrecte d'une voyelle permettant un parler plus fluide. Ce parler est indéniablement caractéristique des jeunes d'aujourd'hui qui affectionnent tout particulièrement ce type de langage. Ils s'y identifient nécessairement ; le publiciste atteint donc son premier objectif consistant à la captation de l'attention de ses futurs clients. Par ailleurs, nous soulignons la présence du terme anglophone francisé « checké », appartenant désormais au langage des jeunes qui se trouve traduit en bas à droite de l'écran par le mot « vérifié » comme le stipule la Loi N94/665 du 4 août 1994 relative à l'emploi de la langue française, appelée plus communément loi Toubon ${ }^{6}$. Ce néologisme né de l’emprunt du verbe « check » à la langue anglaise est révélateur du public que les créateurs de publicité souhaitent approcher : les jeunes français de notre société de consommation, friands de ce nouveau type de langage qui laisse place à la création linguistique. Ces transgressions langagières se trouvent ainsi en tout lieu dans le panorama lexicographique actuel, en grande partie de par le poids considérable des nouvelles technologies et des réseaux sociaux usant d'anglicismes dans la vie de ces citoyens à la page. Nous remarquons d’ailleurs la présence du hashtag, marqueur fréquemment employé sur l'Internet permettant de mettre en relation plusieurs individus sur un thème commun à partir d'un mot-clé, à la fin du spot publicitaire. «\#CmonYop » indique ainsi aux futurs jeunes clients qu’ils peuvent aisément commenter et partager leurs expériences avec ce produit sur les réseaux sociaux, dont on retrouve également les logos apposés au slogan cité précédemment. Enfin, nous nous devons de mettre en relief le fait que le nom du produit en lui-même est un néologisme issu de la troncation du nom de la marque : le terme Yop a en effet été construit grâce à l'apocope de la marque Yoplait. Le lexique employé dans cette vidéo publicitaire s'adapte donc totalement au public cible grâce à l'usage de tournures branchées, évocatrices, transgressives, captivantes, actuelles et motivantes.

\section{Conclusion}

En guise de conclusion, nous pouvons affirmer que le spot publicitaire analysé est représentatif des attentes linguistiques et socio-affectives des adolescents de nos sociétés contemporaines. Il contemple en effet un grand nombre de paramètres sociologiques et émotionnels auxquels les jeunes donnent de l’importance et met en place un langage juvénile et branché au service de mécanismes linguistiques publicitaires persuasifs. Nous rajouterons ici le fait que le site web d'hébergement de vidéo YouTube sur lequel nous avons répertorié cette publicité permet aux utilisateurs d’évaluer et de commenter les contenus visualisés, ce qui a pour résultat de la publicité de publicité.

\section{Références bibliographiques}

BAUDRILlARD, Jean (1970, 1986). La société de consommation. Paris : Gallimard.

BERnHeIM, François (2004). Guide de la publicité et de la consommation. Paris : Larousse.

BRUNE, François (1985). Le bonheur conforme. Paris : Gallimard.

ClaEs, Michel (2003). L’univers social des adolescents. Montréal : Les Presses de l’Université de Montréal.

CosLin, Pierre (2007). La socialisation de l'adolescent. Paris : Armand Colin.

EguizÁBAL, Raúl (2009). Industrias de la consciencia: una historia social de la publicidad en España (1975-2009). Barcelona : Península.

EGUIZÁBAL, Raúl (2007). Teoría de la publicidad. Madrid : Cátedra.

Foucault, Michel (1984). «Des espaces autres » dans Architecture, mouvement, continuité, nº5, p. 46-49. < http://desteceres.com/heterotopias.pdf> [Consulté le 04/04/2016].

Freud, Sigmund $(1923,1968)$. « Le Moi et le Ça » dans Essais de Psychanalyse. Paris : Payot, p. 177-234.

FREUD, Sigmund (1917, 2004). Introduction à la psychanalyse. Paris : Payot.

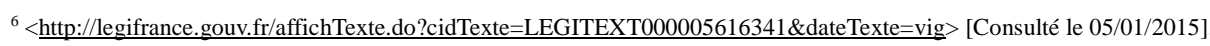


FReUd, Sigmund (1905, 2010). Trois essais sur la théorie sexuelle. Paris : PUF.

JEAN, Sylvie et al. (2014). " Modélisation des comportements de consommation », dans Gillet, Roland. Introduction au marketing. Montreuil : Pearson.

LAGEISTE, Jérôme (2008). « La plage, un objet géographique de désir » dans Rieucau, Jean et Lageiste, Jérôme (sous la direction de). La plage : un territoire atypique. Géographie et culture, nº67. Paris : L’Harmattan, p. 7-26.

LAzZARotTi, Olivier (2012). «Paulette à la mer ou de l'imaginaire géographique comme construction du regard de l'autre » dans Bédard, Mario, Augustin, Jean-Pierre et Desnoilles, Richard (sous la direction de). L'imaginaire géographique : perspectives, pratiques et devenirs. Montréal : Presse Universitaire du Québec, p. 175-192.

LEÓN, José Luis (2001). Mitoanálisis de la publicidad. Barcelona : Ariel.

ORTEGA, Enrique (1997, 2004). La comunicación publicitaria. Madrid : Pirámide, 2ème éd.

SАвот, Philippe (2012). « Langage, société, corps. Utopies et hétérotopies chez Michel Foucault » dans Cremonesi, Laura et al. (sous la direction de). materiali foucaultiani, Vol 1, nº 1, p. 17-35. <https://halshs.archives-ouvertes.fr/halshs-00746742/document> [Consulté le 04/04/2016]. 